\title{
Reduction in Blood Pressure with a Sodium-Reduced, Potassium- and Magnesium-Enriched Mineral Salt in Subjects with Mild Essential Hypertension
}

\author{
Terukazu Kawasaki, Kazue Itoh*, and Masumi Kawasaki
}

\begin{abstract}
A parallel controlled clinical trial was carried out to investigate the effect on blood pressure (BP) of replacing normal salt with mineral salt in seasonings. After a 2-wk run-in period, 21 subjects (10 men and 11 women; age, $66.0 \pm 7.6 \mathrm{yr}$ ) were given mineral salt in seasonings instead of normal salt [mineral salt (MS) group], while 20 subjects (10 men and 10 women; age, $65.9 \pm 7.4 \mathrm{yr}$ ) continued to receive normal salt in seasonings [regular salt (RS) group] for the next $5 \mathrm{wk}$ in the experimental (E) period. In the MS group, systolic (S) and diastolic (D) BP decreased significantly from $134.7 \pm 17.2 / 77.2 \pm 9.7$ at baseline (week 0) to $127.3 \pm 12.0 / 73.5 \pm 8.9 \mathrm{mmHg}$ at the end of the $E$ period (week 5), and the reductions in both SBP and DBP were larger in hypertensive subjects than in normotensive subjects in the MS group. The serum sodium and chloride concentrations decreased significantly, while the serum potassium and HDL-cholesterol concentrations increased significantly at week 5. The 24-h urinary sodium excretion decreased, and the 24-h potassium and magnesium excretions increased significantly from week 0 to week 5 in the MS group. In contrast, SBP, DBP, serum chemistry, and urinary electrolyte excretion did not change significantly in the RS group. These findings suggest that the excessive salt content and insufficient potassium and magnesium content of the present Japanese diet could be easily and safely corrected by replacing seasonings prepared with regular salt with those prepared with mineral salt. (Hypertens Res 1998; 21: 235-243)
\end{abstract}

Key Words: dietary sodium, potassium supplement, magnesium supplement, mineral salt, blood pressure, hypertension

Numerous studies have suggested that sodium may play an important role in the development and maintenance of hypertension (1-9) with a few exceptions $(10,11)$. Although a reduction in sodium intake may lower blood pressure (BP), recent studies indicate that the antihypertensive effects of a low-sodium diet may be enhanced by simultaneous potassium supplementation (12). Lowering the sodium intake or increasing the potassium intake, or both, have been shown to exert a beneficial effect on high BP (13-15). There is also evidence that an increased intake of magnesium may favorably affect BP (16-20). Such evidence has thus led to recommendations by the Joint National Committee on Detection, Evaluation and Treatment of High Blood Pressure (21) to restrict the intake of salt to less than $6 \mathrm{~g}$ per $\mathrm{d}$, while additional recommendations of the committee include maintaining an adequate dietary intake of potassium, magnesium, and calcium (22).

These observations thus indicate that a modest reduction in sodium intake combined with an increased intake of potassium and magnesium might have therapeutic effects in essential hypertensive subjects. In particular, elderly hypertensive subjects tend to be salt sensitive and more responsive to sodium restriction than younger subjects (23). However, many subjects with hypertension find it difficult to give up the taste of regular salt and consequently are unable to sufficiently reduce their intake to have an effect on BP, even though sodium restriction is widely recognized as the best non-pharmacological approach to the prevention and treatment of hypertension $(21,22)$. Compliance with a low-salt diet is particularly difficult for elderly Japanese subjects because their diet is high in salt and includes salt-containing products such as soy sauce and miso.

A new reduced-sodium-content mineral salt containing potassium and magnesium, which was originally produced by Karppanen $(24,25)$, may aid in the prevention and management of hypertension while also offering consumers a tasty alternative to regular salt. To explore the antihypertensive effects of mineral salt, we performed a trial of mild dietary sodium reduction with mild potassium and magne-

From the Institute of Health Science, Kyushu University, Kasuga, Japan, and *Nakamura Gakuen University, Fukuoka, Japan.

Address for Reprints: Terukazu Kawasaki, M.D., Ph.D., Institute of Health Science, Kyushu University, Kasuga 816-8580, Japan.

Received November 29, 1997; accepted in revised form September 28, 1998. 
Table 1. Clinical Characteristics of the Subjects at Entry

\begin{tabular}{lcc}
\hline & Regular Salt Group & Mineral Salt Group \\
\hline Number of subjects & 20 & 21 \\
Sex (males/females) & $10 / 10$ & $10 / 11$ \\
Age (yr) & $65.8 \pm 7.6$ & $65.9 \pm 7.4$ \\
Height $(\mathrm{cm})$ & $157.6 \pm 9.3$ & $156.6 \pm 8.3$ \\
Weight $(\mathrm{kg})$ & $57.8 \pm 7.8$ & $56.0 \pm 7.3$ \\
Body mass index $\left(\mathrm{kg} / \mathrm{m}^{2}\right)$ & $23.3 \pm 2.3$ & $22.9 \pm 2.7$ \\
Systolic blood pressure $(\mathrm{mmHg})$ & $133.2 \pm 18.9$ & $136.9 \pm 16.6$ \\
Diastolic blood pressure $(\mathrm{mmHg})$ & $77.2 \pm 12.2$ & $76.6 \pm 9.4$ \\
Pulse rate (beats/min) & $70.1 \pm 10.5$ & $71.7 \pm 10.4$ \\
\hline
\end{tabular}

sium supplementation in normotensive and hypertensive Japanese middle-aged and elderly subjects. An additional study including healthy subjects was also conducted to examine the effects on taste of mineral salt and regular salt in seasonings.

\section{Subjects and Methods}

Subjects

More than 50 of approximately 200 clinically healthy middle-aged and elderly male and female volunteers with ordinary life styles in a certain community responded to a questionnaire that stated in detail the objectives and clinical relevance of the study. Individuals were excluded from the study if they planned to travel during the study period, frequently dined away from home, or had a high risk of noncompliance with the protocol. Finally, 41 subjects were enrolled; written informed consent was obtained from all subjects. These subjects were randomly assigned to receive either mineral salt (MS group) or regular salt (RS group). Both groups were similar with respect to age, sex, body size, and blood pressure during the run-in period. Four subjects in each group who were receiving antihypertensive drugs $(\beta$-blockers, calcium-channel blockers, or both) were requested not to change the dose or administration schedule during the study, including the run-in period. Although no special limitations were placed on daily activities, the subjects were instructed to refrain from taking day trips and from eating away from home. If they had to dine out, they were requested to take with them lunch boxes prepared using the mineral salt seasoning whenever possible. The protocol was approved by the Ethics Committee of the Japan Nutrition Society before starting the study.

Table 1 shows the baseline clinical characteristics of the subjects enrolled in the study. There was no significant difference in any variable between the two groups. Each group included both normotensive (NT: SBP $<140 \mathrm{mmHg}$ and $\mathrm{DBP}<90 \mathrm{mmHg}$ ) and hypertensive subjects (HT: SBP $\geq 140 \mathrm{mmHg}$, DBP $\geq 90 \mathrm{mmHg}$, or both). There were no significant differences in variables other than $\mathrm{BP}$ values between the NT and HT subjects in either the RS or MS group.

\section{Methods \\ Protocol}

The subjects were strictly instructed to avoid changing their ordinary diet during the 7-wk study period. After a 2-wk run-in period, the MS group consumed food containing mineral salt instead of the three seasonings of salt, soy sauce, and miso for the next $5 \mathrm{wk}$, designated as the experimental (E) period. Body weight and BP were measured, and the subjects were instructed again not to alter their life styles $2 \mathrm{wk}$ after starting the $\mathrm{E}$ period. Good compliance was maintained throughout the study.

BP and pulse rate were measured three times consecutively with an automatic sphygmomanometer BP-203 (Nippon Colin Co., Ltd., Komaki, Japan) (26) every 2 min at about 8:00 AM on the first and the last days of the run-in period (week 0 ), and $2 \mathrm{wk}$ (week 2) and $5 \mathrm{wk}$ after starting the E period (week 5), after the subjects had urinated and sat down quietly for at least $10 \mathrm{~min}$. The average of the three measurements was used for analysis.

After measuring BP, blood samples were collected in the early morning after the subjects had fasted for $12 \mathrm{~h}$ and rested for $30 \mathrm{~min}$ in the supine position on the last day of each period. The serum and plasma were separated by centrifugation and stored at $-40^{\circ} \mathrm{C}$ until assay. Urine was collected for $24 \mathrm{~h}$ on the last day of each period. The urinary concentrations of sodium, potassium, magnesium, calcium, and creatinine $(\mathrm{Cr})$ and urine volume were measured and the 24-h excretions were calculated. The completeness of the 24-h urine collection was evaluated on the basis of the creatinine value predicted using our maneuver as described elsewhere (27).

After the run-in period, the subjects in the RS group continued to eat their normal diet containing the same seasonings for the next $5 \mathrm{wk}$. The subjects were requested to continue their usual activities without modification, similar to the subjects in the MS group. Furthermore, we regularly contacted all subjects by telephone to ensure that their life styles were not changed. Blood samples and 24-h urine were collected twice, similar to the MS group.

Determination of electrolyte content of seasonings

The contents of sodium, potassium, and magnesium in $100 \mathrm{~g}$ of three different seasonings, (table salt, soy sauce, and miso) prepared with regular salt and 
Table 2. Comparison of Electrolyte Contents of $100 \mathrm{~g}$ of Table Salt, Soy Sause, and Miso Prepared with Regular Salt or Mineral Salt

\begin{tabular}{llrrr}
\hline Electrolytes & & Sodium & Potassium & Magnesium \\
\hline Table salt & Regular salt & 39,000 & 130 & 21 \\
& Mineral salt & 22,900 & 10,110 & 1,240 \\
Soy sauce & Ratio & 0.59 & 77.8 & 59.1 \\
& Regular salt & 5,400 & 310 & 69 \\
& Mineral salt & 4,250 & 1,230 & 160 \\
Miso & Ratio & 0.79 & 3.97 & 2.32 \\
& Regular salt & 4,280 & 215 & 46 \\
& Mineral salt & 2,850 & 1,640 & 175 \\
& Ratio & 0.67 & 7.63 & 3.80 \\
\hline
\end{tabular}

Unit: $\mathrm{mg} / 100 \mathrm{~g}$, Ratio: Ratio of electrolyte content of regular salt to that of mineral salt.

mineral salt were measured at our laboratory. Table 2 shows the electrolyte content of $100 \mathrm{~g}$ of regular salt and mineral salt and the electrolyte content of $100 \mathrm{~g}$ of soy sauce and miso prepared with regular salt and mineral salt. The sodium content of the mineral salt used during the $\mathrm{E}$ period was $59 \%$ that of the salt used during the run-in period, and the potassium and magnesium contents of the mineral salt were 78 times and 59 times higher than those of regular salt, respectively. Although the sodium content of the mineral salt soy sauce used during the $\mathrm{E}$ period was $79 \%$ that of the soy sauce used during the run-in period, the potassium and magnesium contents of the former were, respectively, 4 times and 2.3 times those of the latter. In addition, the sodium content of the mineral salt miso during the E period was $67 \%$ that of the miso used during the run-in period, whereas the potassium and magnesium contents of the former were 7.6 times and 3.8 times higher than those of the latter.

The blind sensory taste study

Twenty-four healthy subjects $(10$ men and 14 women) 20 to $75 \mathrm{yr}$ of age who had normal life styles participated in the taste test. The test foods were presented randomly to avoid bias. The subjects were asked to consume both a miso soup and a clear soup made with soy sauce on different occasions and to evaluate the saltiness, palatability, and savoriness of each.

The control food was a clear soup containing Kikkoman soy sauce ${ }^{\circledR}$ and commercially sold salt, while the test food was a clear soup containing the same amounts of mineral salt soy sauce and mineral salt as control. Blind sensory tests were conducted using four different clear soups: regular salt $[\mathrm{A}]$ and mineral salt $[\mathrm{B}]$ with $0.8 \%$ salinity, and regular salt $[C]$ and mineral salt [D] with $1.0 \%$ salinity. Sensory tests for the miso soups were conducted with a soup containing Marukome miso ${ }^{\circledR}$, made with regular salt, and a soup containing the same amount of 'mineral salt miso'.

In both sensory taste tests, the total scores were obtained by the rank method and were tested with Kendall's coefficient of concordance. As concordance was confirmed, the mean scores were compared by the rank method. The lower score repre- sented better saltiness, palatability, or savoriness.

In the blind sensory taste test with four kinds of clear soups, A (regular salt: $0.8 \%$ ), B (mineral salt: $0.8 \%$ ), C (regular salt: $1.0 \%$ ), and D (mineral salt: $1.0 \%$ ), total scores were obtained by the rank method and were tested with Kendall's coefficient of concordance. As concordance was confirmed, the mean scores were compared by the rank method. The best score was 1 point and the worst 4 . The lower scores for salty taste, palatability, and savoriness thus represented better or the more preferred quality.

Salty taste was weakest for soup B and strongest for soup C. There was no difference between soup A and soup B or D. As for palatability and savoriness, clear soup $\mathrm{A}$ was best. There were no differences in palatability between soup A and D, and no difference in savoriness among the four soups (Table 3).

Blind sensory taste tests were conducted with miso soups prepared with equivalent amounts of miso $(10 \%)$ containing either regular salt or mineral salt. As shown in Table 3, a significant difference was found only in salty taste; soup E was felt to be more salty than the other soups. There were no differences in palatability or savoriness between miso soups $E$ and $F$.

There were no significant differences in saltiness, palatability, and savoriness of either the clear soups or miso soups between subjects younger than $60 \mathrm{yr}$ of age and those $60 \mathrm{yr}$ of age or older.

\section{Laboratory and Statistical Methods}

The concentrations of sodium and potassium in serum and urine were measured by flame photometry (Hitachi 775, Hitachi Co., Ltd., Hitachi, Japan), and the concentration of $\mathrm{Cr}$ in serum and urine was measured by the picric acid method (Olympus AU5000, Olympus Optical Co., Ltd., Tokyo, Japan). Serum chemistry was analyzed with autoanalyzers (Olympus AU-5000 and AUA-8000, Olympus Optical Co., Ltd., Tokyo, Japan). Serum and urinary magnesium and calcium concentrations were measured by atomic absorption spectrophotometry (Shimadzu AA660, Shimadzu Co., Ltd., Tokyo, Japan). LDL-cholesterol was calculated by the formula of 
Table 3. Mean Scores by the Rank Method in a Blind Sensory Test to Evaluate Salty Taste, Palatability, and Savoriness

\begin{tabular}{llll}
\hline & Salty taste & Palatability & Savoriness \\
\hline A. Clear soup with regular salt $(0.8 \%)^{\mathrm{a})}$ & $2.44 \pm 0.83$ & $2.00 \pm 1.00$ & $2.36 \pm 1.05$ \\
B. Clear soup with mineral salt $(0.8 \%)^{\mathrm{b})}$ & $1.83 \pm 1.24$ & $2.87 \pm 1.10^{* *}$ & $2.59 \pm 1.18$ \\
C. Clear soup with regular salt $(1.0 \%)^{\mathrm{a})}$ & $3.15 \pm 1.14^{*}$ & $2.70 \pm 1.15^{*}$ & $2.64 \pm 1.14$ \\
D. Clear soup with mineral salt $(1.0 \%)^{\mathrm{b})}$ & $2.58 \pm 0.88$ & $2.43 \pm 1.16$ & $2.41 \pm 1.14$ \\
\hline E. Miso soup with regular salt ${ }^{\mathrm{b}}$ & $1.92 \pm 0.27$ & $1.48 \pm 0.51$ & $1.38 \pm 0.50$ \\
F. Miso soup with mineral salt & $1.08 \pm 0.27^{\dagger \dagger}$ & $1.52 \pm 0.51$ & $1.62 \pm 0.50$ \\
\hline
\end{tabular}

${ }^{*} p<0.01,{ }^{* *} p<0.01$ (vs. A); ${ }^{\dagger \dagger} p<0.01$ (vs. E).

a): $0.8 \%$ or $1.0 \%$ salinity prepared with Kikkoman soy sauce and commercially available salt, b): $0.8 \%$ or $1.0 \%$ salinity prepared with mineral salt soy sauce and mineral salt, c): Miso soup prepared with Marukome miso using commercially available salt, d): Miso soup prepared with the same amount of miso containing mineral salt.

Systolic BP

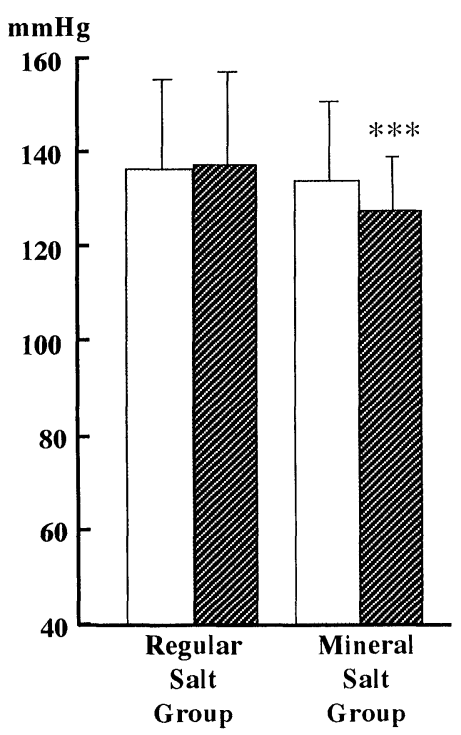

Diastolic BP

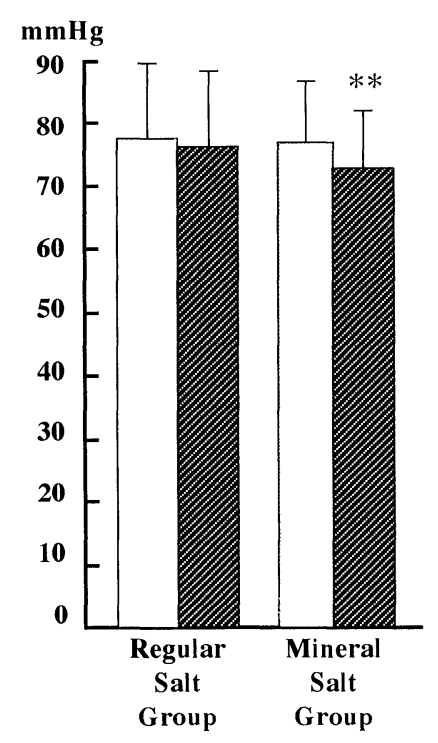

Pulse

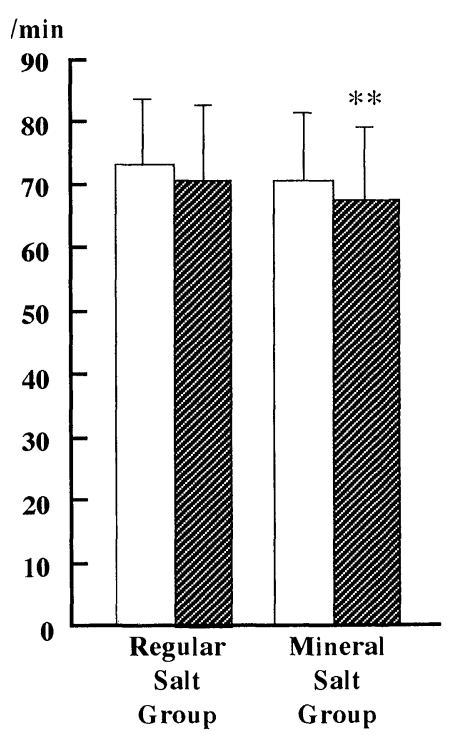

Fig. 1. Changes in systolic and diastolic blood pressure (BP) and pulse rate from baseline (week 0: $\square$ ( ing regular salt $(\mathrm{n}=20)$ and mineral salt $(\mathrm{n}=21)$ in seasonings and certain processed foods for 5 wk (week 5 : Values are means $\pm S D,{ }^{* *} \mathrm{p}<0.01, * * * \mathrm{p}<0.001$ (vs. week 0 )

Friedewald et al. (28). The plasma renin activity (PRA), plasma aldosterone concentration (PAC), and urinary aldosterone excretion (AER) were determined by a radioimmunoassay. The tests were conducted simultaneously for each subject's serum and urine samples.

All results are expressed as means \pm standard deviations (SD). Any differences between the run-in and E period were evaluated by Student's paired $t$ test for variables showing an approximately normal distribution. Nonparametric analyses using the Mann Whitney $U$-test were performed for data not normally distributed. A value of $p<0.05$ was considered to indicate statistical significance.

\section{Results}

Changes in the SBP, DBP, and pulse rate from baseline (week 0) to after receiving the mineral salt in seasonings and selected processed foods for $5 \mathrm{wk}$ (week 5) are shown in Fig. 1. In the MS group, both SBP and DBP decreased significantly from $134.7 \pm 17.2 \mathrm{mmHg}$ at week 0 to $125.8 \pm 15.9$ at week 2 and $127.3 \pm 12.0$ at week 5 and from $77.2 \pm$ $9.7 \mathrm{mmHg}$ to $72.9 \pm 9.6$ and $73.5 \pm 8.9$, respectively. Pulse rate also decreased significantly from 71.0 \pm 10.8 beats $/ \mathrm{min}$ at week 0 to $70.5 \pm 9.7$ at week 2 and $67.5 \pm 11.1$ at week 5 in the MS group. In contrast, there were no significant changes in SBP, DBP, or pulse rate in the RS group (Fig. 1). The reductions in both SBP and DBP were larger in the $10 \mathrm{HT}$ subjects (from $149.1 \pm 10.9 \mathrm{mmHg}$ to 136.8 \pm 9.6 and $133.7 \pm 8.3$ for SBP and from $85.6 \pm 5.9$ $\mathrm{mmHg}$ to $80.9 \pm 5.2$ and $79.3 \pm 6.8$ for DBP) than in the $11 \mathrm{NT}$ subjects (from $121.7 \pm 6.0 \mathrm{mmHg}$ to $115.7 \pm 13.8$ and $121.5 \pm 12.2$ for SBP and from 
$69.5 \pm 4.3 \mathrm{mmHg}$ to $65.6 \pm 6.0$ and $68.3 \pm 7.3$ for DBP) in the MS group (Fig. 2).

Body weight did not change at all in either the MS $(56.0 \pm 7.3 \mathrm{~kg}$ at week 0 vs. $56.0 \pm 7.3 \mathrm{~kg}$ at

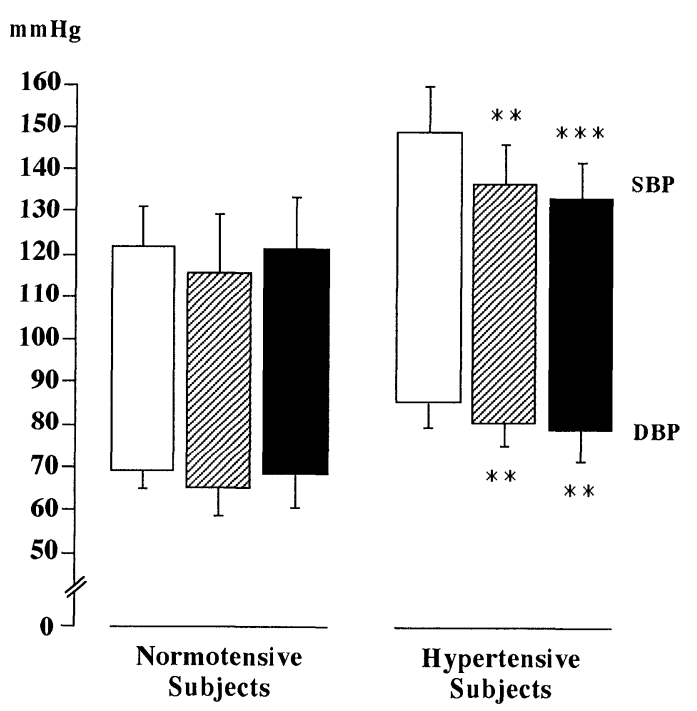

Fig. 2. Changes in systolic (SBP) and diastolic blood pressure $(D B P)$ from baseline (week $0: \square$ : to after receiving mineral salt in seasonings and certain processed foods for 2 wk (week 2: VIIIIIIIIIX) and 5 wk (week 5: $\square$ in normotensive and hypertensive subjects. Values are means $\pm S D, * \mathrm{p}<0.05,{ }^{* *} \mathrm{p}<0.01,{ }^{* * *} \mathrm{p}<0.001$ (vs. week 0). week 5) or RS group (57.8 $\pm 7.9 \mathrm{~kg}$ vs. $57.9 \pm 8.2$ $\mathrm{kg}$ ) during the $\mathrm{E}$ period.

The 24-h urinary electrolyte excretion on the last day of each period are shown according to NT and HT subjects in each group in Table 4. The 24-h urinary sodium excretion in both NT and HT subjects in the MS group slightly but insignificantly decreased from week 0 to week 5 . When NT and HT subjects were combined, the reduction in the $24-\mathrm{h}$ urinary sodium excretion was statistically significant between week 0 and week 5 . The 24-h urinary potassium and magnesium excretion increased significantly from $51.5 \mathrm{mEq}$ and $9.9 \mathrm{mg}$ at week 0 to $69.0 \mathrm{mEq}$ and $11.6 \mathrm{mg}$ at week 5 in the MS group. A significant increase in urinary potassium excretion was observed in both NT and HT subjects, while urinary magnesium excretion increased significantly only in HT subjects. The 24-h urinary calcium and $\mathrm{Cr}$ excretion did not change. Urinary electrolyte excretion changed slightly but not significantly in both NT and HT subjects in the RS group.

Serum electrolytes and lipids were measured at week 0 and week 5 (Table 5). The serum sodium and chloride concentrations decreased and the serum potassium and HDL-cholesterol concentrations increased significantly after $5 \mathrm{wk}$ of mineral salt consumption. When the data were analyzed according to NT and HT subjects in each group, however, there was no significant change in serum sodium or chloride concentration in NT subjects or in serum HDL-cholesterol concentration in either HT or NT subjects. LDL-cholesterol concentration and atherosclerosis index (total cholesterol/HDLcholesterol) did not change after mineral salt inges-

Table 4. Urinary Excreation of Sodium, Potassium, Magnesium, Calcium, and Creatinine in Normotensive and Hypertensive Subjects at Baseline (Week 0) and After Receiving Either Regular Salt or Mineral Salt in Seasonings for 5 Weeks (Week 5)

Regular Salt Group

\begin{tabular}{|c|c|c|c|c|c|c|}
\hline & \multicolumn{2}{|c|}{ All Subjects $(n=20,10 \mathrm{M} / 10 \mathrm{~F})$} & \multicolumn{2}{|c|}{ Normotensives $(n=12,7 \mathrm{M} / 5 \mathrm{~F})$} & \multicolumn{2}{|c|}{ Hypertensives $(n=8,3 \mathrm{M} / 5 \mathrm{~F})$} \\
\hline & Week 0 & Week 5 & Week 0 & Week 5 & Week 0 & Week 5 \\
\hline Urine $\mathrm{Na}(\mathrm{mEq} / \mathrm{d})$ & $171 \pm 47$ & $182 \pm 50$ & $174 \pm 57$ & $177 \pm 44$ & $174 \pm 50$ & $185 \pm 50$ \\
\hline Urine $\mathrm{K}(\mathrm{mEq} / \mathrm{d})$ & $56.9 \pm 17.3$ & $61.5 \pm 18.2$ & $53.8 \pm 15.5$ & $60.2 \pm 18.3$ & $55.0 \pm 21.4$ & $62.7 \pm 21.2$ \\
\hline Urine $\mathrm{Mg}(\mathrm{mg} / \mathrm{d})$ & $11.6 \pm 6.1$ & $11.6 \pm 6.8$ & $11.4 \pm 6.6$ & $11.7 \pm 5.9$ & $11.9 \pm 5.8$ & $11.4 \pm 8.4$ \\
\hline Urine $\mathrm{Ca}(\mathrm{mg} / \mathrm{d})$ & $10.1 \pm 4.0$ & $10.4 \pm 4.4$ & $9.4 \pm 3.3$ & $10.0 \pm 4.1$ & $10.9 \pm 4.9$ & $10.9 \pm 5.2$ \\
\hline Urine volume $(\mathrm{ml} / \mathrm{d})$ & $1,560 \pm 673$ & $1,600 \pm 570$ & $1,477 \pm 543$ & $1,510 \pm 385$ & $1,696 \pm 855$ & $1,738 \pm 784$ \\
\hline Urine $\mathrm{Cr}(\mathrm{mg} / \mathrm{d})$ & $965 \pm 252$ & $966 \pm 257$ & $1,023 \pm 289$ & $1,023 \pm 297$ & $878 \pm 161$ & $881 \pm 161$ \\
\hline
\end{tabular}

Mineral Salt Group

\begin{tabular}{|c|c|c|c|c|c|c|}
\hline & \multicolumn{2}{|c|}{ All Subjects $(n=21,10 \mathrm{M} / 11 \mathrm{~F})$} & \multicolumn{2}{|c|}{ Normotensives $(n=11,6 \mathrm{M} / 5 \mathrm{~F})$} & \multicolumn{2}{|c|}{ Hypertensives $(n=10,4 \mathrm{M} / 6 \mathrm{~F})$} \\
\hline & Week 0 & Week 5 & Week 0 & Week 5 & Week 0 & Week 5 \\
\hline Urine $\mathrm{Na}(\mathrm{mEq} / \mathrm{d})$ & $192 \pm 61$ & $173 \pm 56^{*}$ & $174 \pm 63$ & $158 \pm 61$ & $211 \pm 55$ & $189 \pm 49$ \\
\hline Urine K (mEq/d) & $51.5 \pm 12.6$ & $69.0 \pm 19.1^{* * *}$ & $51.7 \pm 14.3$ & $63.9 \pm 23.2 *$ & $52.3 \pm 10.7$ & $72.4 \pm 10.1^{* * *}$ \\
\hline Urine $\mathrm{Mg}(\mathrm{mg} / \mathrm{d})$ & $9.9 \pm 2.8$ & $11.6 \pm 4.4 * *$ & $9.5 \pm 2.7$ & $11.2 \pm 5.1$ & $10.3 \pm 3.1$ & $12.0 \pm 3.8 *$ \\
\hline Urine $\mathrm{Ca}(\mathrm{mg} / \mathrm{d})$ & $9.2 \pm 4.1$ & $9.2 \pm 3.7$ & $11.2 \pm 5.1$ & $13.4 \pm 6.1$ & $8.7 \pm 5.3$ & $7.5 \pm 4.0$ \\
\hline Urine volume $(\mathrm{ml} / \mathrm{d})$ & $1,600 \pm 453$ & $1,550 \pm 453$ & $1,515 \pm 389$ & $1,528 \pm 506$ & $1,704 \pm 527$ & $1,586 \pm 407$ \\
\hline Urine $\mathrm{Cr}(\mathrm{mg} / \mathrm{d})$ & $908 \pm 226$ & $949 \pm 248$ & $891 \pm 242$ & $946 \pm 232$ & $927 \pm 217$ & $945 \pm 274$ \\
\hline
\end{tabular}

${ }^{*} p<0.05,{ }^{* *} p<0.01,{ }^{* * *} p<0.001$ (vs. Week 0) 
Table 5. Serum Chemistry in Normotensive and Hypertensive Subjects at Baseline (Week 0) and After Receiving Either Regular Salt or Mineral Salt in Seasonings for 5 Weeks (Week 5)

Regular Salt Group

\begin{tabular}{|c|c|c|c|c|c|c|}
\hline & \multicolumn{2}{|c|}{ All Subjects $(n=20,10 \mathrm{M} / 10 \mathrm{~F})$} & \multicolumn{2}{|c|}{ Normotensives $(n=12,7 \mathrm{M} / 5 \mathrm{~F})$} & \multicolumn{2}{|c|}{ Hypertensives $(n=8,3 \mathrm{M} / 5 \mathrm{~F})$} \\
\hline & Week 0 & Week 5 & Week 0 & Week 5 & Week 0 & Week 5 \\
\hline Sodium $(\mathrm{mEq} / \mathrm{l})$ & $141.2 \pm 1.4$ & $141.0 \pm 1.3$ & $140.8 \pm 1.4$ & $140.7 \pm 1.6$ & $141.8 \pm 1.2$ & $141.4 \pm 0.5$ \\
\hline Potassium (mEq/l) & $4.44 \pm 0.37$ & $4.46 \pm 0.34$ & $4.4 \pm 0.4$ & $4.5 \pm 0.3$ & $4.4 \pm 0.4$ & $4.4 \pm 0.4$ \\
\hline Chloride $(\mathrm{mEq} / \mathrm{l})$ & $101.8 \pm 2.4$ & $102.5 \pm 2.3$ & $102.2 \pm 2.6$ & $103.2 \pm 1.9$ & $101.3 \pm 2.3$ & $101.5 \pm 2.5$ \\
\hline Magnesium (mg/dl) & $2.34 \pm 0.15$ & $2.33 \pm 0.16$ & $2.3 \pm 0.1$ & $2.4 \pm 0.2$ & $2.3 \pm 0.2$ & $2.3 \pm 0.1$ \\
\hline Total cholesterol (mg/dl) & $227.5 \pm 40.2$ & $227.6 \pm 29.3$ & $212.1 \pm 17.2$ & $221.4 \pm 24.2$ & $250.6 \pm 53.8$ & $236.8 \pm 35.2$ \\
\hline HDL-cholest. (mg/dl) & $54.7 \pm 14.6$ & $54.4 \pm 13.9$ & $51.2 \pm 12.4$ & $50.3 \pm 12.3$ & $60.0 \pm 16.6$ & $60.5 \pm 14.7$ \\
\hline Triglyceride $(\mathrm{mg} / \mathrm{dl})$ & $130.9 \pm 75.4$ & $137.0 \pm 90.1$ & $135.4 \pm 93.1$ & $144.0 \pm 105.3$ & $116.4 \pm 47.4$ & $137.4 \pm 63.7$ \\
\hline
\end{tabular}

Mineral Salt Group

\begin{tabular}{|c|c|c|c|c|c|c|}
\hline & \multicolumn{2}{|c|}{ All Subjects $(n=21,10 \mathrm{M} / 11 \mathrm{~F})$} & \multicolumn{2}{|c|}{ Normotensives $(n=11,6 \mathrm{M} / 5 \mathrm{~F})$} & \multicolumn{2}{|c|}{ Hypertensives $(n=10,4 \mathrm{M} / 6 \mathrm{~F})$} \\
\hline & Week 0 & Week 5 & Week 0 & Week 5 & Week 0 & Week 5 \\
\hline Sodium $(\mathrm{mEq} / \mathrm{l})$ & $140.9 \pm 1.4$ & $139.6 \pm 1.8^{* *}$ & $140.8 \pm 1.4$ & $140.1 \pm 1.5$ & $140.9 \pm 1.5$ & $139.1 \pm 2.0 * *$ \\
\hline Potassium (mEq/l) & $4.33 \pm 0.39$ & $4.71 \pm 0.34 * * *$ & * $\quad 4.28 \pm 0.48$ & $4.64 \pm 0.38 *$ & $4.39 \pm 0.25$ & $4.80 \pm 0.27 * * *$ \\
\hline Chloride (mEq/l) & $103.4 \pm 2.1$ & $102.5 \pm 2.3 * *$ & $103.8 \pm 1.8$ & $103.3 \pm 2.2$ & $102.9 \pm 2.4$ & $101.7 \pm 2.2 *$ \\
\hline Magnesium (mg/dl) & $2.30 \pm 0.13$ & $2.31 \pm 0.13$ & $2.31 \pm 0.09$ & $2.31 \pm 0.13$ & $2.29 \pm 0.18$ & $2.31 \pm 0.13$ \\
\hline Total cholesterol (mg/dl) & $208.9 \pm 29.8$ & $211.0 \pm 29.8$ & $208.6 \pm 29.0$ & $207.8 \pm 29.9$ & $209.2 \pm 32.3$ & $214.4 \pm 31.0$ \\
\hline HDL-cholest. (mg/dl) & $63.7 \pm 18.4$ & $65.6 \pm 17.9 * *$ & $65.9 \pm 17.3$ & $68.4 \pm 16.9$ & $61.2 \pm 20.2$ & $63.1 \pm 19.4$ \\
\hline Triglyceride $(\mathrm{mg} / \mathrm{dl})$ & $107.3 \pm 55.1$ & $93.3 \pm 41.4$ & $95.2 \pm 47.3$ & $92.6 \pm 50.3$ & $120.6 \pm 62.2$ & $100.4 \pm 41.7$ \\
\hline
\end{tabular}

${ }^{*} p<0.05,{ }^{* *} p<0.01,{ }^{* * *} p<0.001$ ( $v s$. Week 0 )

tion in either NT or HT subjects in the MS group.

Changes in PRA and AER were compared between week 0 and week 5 . In the MS group, PRA did not change significantly (from $0.43 \pm 0.32$ to $0.60 \pm 0.51 \mathrm{ng} / \mathrm{ml} / \mathrm{h}$ ), whereas both PAC and AER increased significantly during the $\mathrm{E}$ period from 7.6 \pm 2.9 to $10.4 \pm 5.4 \mathrm{ng} / \mathrm{dl}(p<0.01)$ and from 12.2 \pm 5.6 to $15.8 \pm 10.0 \mu \mathrm{g} / \mathrm{d}(p<0.05)$, respectively. However, no significant changes in these variables were found in the RS group.

\section{Discussion}

The Japanese diet is high in salt and salt-containing seasonings such as soy sauce and miso. A survey on National Nutrition in Japan (1995) revealed that the average daily intake of salt $(\mathrm{NaCl})$ was $13.2 \mathrm{~g}$. The use of seasonings prepared with the mineral salt, a novel potassium-, magnesium-, and $l$-lysine-enriched salt alternative, can reduce salt intake by $21 \%(29)$. The additional use of certain processed foods made with mineral salt is thus expected to further reduce salt intake.

In some previous investigations, a modest reduction in sodium intake combined with increased intake of potassium, magnesium, or both was reported to have an antihypertensive effect in patients with essential hypertension $(18,24)$ or hypertensive Type II diabetes mellitus (30). We therefore studied the effects of using either regular salt or mineral salt in seasonings and certain processed foods in middleaged and elderly subjects with normotension and mild hypertension over a 5-wk period. We found that both SBP and DBP fell significantly in the MS group as compared with baseline in the HT subjects. The 24-h urinary sodium excretion decreased and potassium and magnesium excretion increased significantly, accompanied by increases in plasma and urinary aldosterone concentrations but no change in plasma renin activity in the HT subjects. These findings suggest that a reduced sodium intake combined with an increased intake of potassium and magnesium, as evidenced by the changes in serum and urine variables, helps to lower blood pressure in Japanese patients with essential hypertension. Although a high sodium intake is thought to contribute to hypertension, elevated blood pressure does not develop in all individuals exposed to a high-salt diet. Approximately one third to $40 \%$ of hypertensive patients are estimated to be salt-sensitive $(2,3$, 31). Most investigators agree that sodium intake can be decreased without harm and that this will help lower blood pressure. There is also increasing evidence that a low intake of potassium (9) and magnesium (32) may contribute to BP elevation.

The mechanism responsible for the reduction in blood pressure in our study is poorly understood. Modest sodium restriction alone has been shown to lower blood pressure in hypertensive subjects in several previous studies (33-37). In the present study, urinary sodium excretion decreased significantly after $5 \mathrm{wk}$ of receiving mineral salt in the MS group. Other previous studies (12-18), including ours $(19,20)$, have consistently demonstrated an antihypertensive effect of dietary potassium and magnesium supplementation in patients with essen- 
tial hypertension. Moreover, epidemiologic evidence also supports an inverse relation between potassium intake and BP. In a meta-analysis of 33 clinical trials involving 1,560 hypertensive and 1,005 normotensive subjects, oral potassium supplementation was shown to significantly lower both SBP and DBP (12).

In a previous study, we found a significant negative correlation between the percent change in urinary norepinephrine (NE) excretion from week 0 to week 2 or week 4 and the corresponding changes in urinary magnesium excretion. There was also a positive correlation between the percent change in urinary NE excretion from week 0 to week 4 and that in DBP $(19,20)$. These results suggest that oral magnesium supplementation might lower BP by decreasing adrenergic activity, which may be involved in the urinary excretion of NE. These findings also suggest that a natriuretic phenomenon may occur through the activation of prostaglandin- $\mathrm{I}_{2}\left(\mathrm{PGI}_{2}\right)$ after magnesium supplementation (38), although $\mathrm{PGI}_{2}$ was not measured in the present study. The vasodilatory activity of magnesium is related to impairment of calcium permeability of the cellular membrane, calcium binding, and translocation in vascular smooth muscle cells (39). Urinary magnesium excretion increased only in HT subjects during the MS period, but there was no change in free $\mathrm{Mg}^{++}$, which is considered to lower BP through its vasodilatory activity. In agreement with the findings of previous studies, the urinary potassium and magnesium excretions increased significantly at week 5 in HT subjects in the MS group in the present study, indicating that mild dietary potassium and magnesium supplementation combined with mild sodium reduction may contribute to decreases in both SBP and DBP in patients with essential hypertension.

A significant increase in the serum HDL-cholesterol concentration was observed at week 5 in the MS group. Only a few studies have evaluated the effects of oral potassium or magnesium supplementation on serum lipid metabolism in humans $(19,20,40)$. In our previous study, serum total cholesterol and triglyceride concentrations decreased significantly after $10 \mathrm{~d}$ of potassium supplementation (19). These favorable effects may be due to a rise in lipoprotein-lipase activity via increased insulin secretion due to potassium supplementation (40). We also found a significant decrease in the serum LDLcholesterol concentration and a significant increase in apo-AI during magnesium supplementation (20). Several studies have reported the effects of magnesium supplementation on serum lipid composition in humans $(30,41,42)$. None of them, however, described the mechanism underlying the effects of magnesium supplementation on hyperlipidemia. We found that serum lecithin-cholesterol acyltransferase (LCAT) activity was increased significantly by magnesium supplementation (20), and to our knowledge no other reports have described a relation between the magnesium level and LCAT activity in humans. We previously reported that the percent change in HDL-cholesterol concentration and that in LCAT showed a significant positive correlation on multiple regression analysis (20), suggesting that the increase in LCAT activity due to mild magnesium supplementation may have contributed to improved lipid metabolism in the MS group.

The sodium/potassium ratio is believed to be more important than the absolute amount of ingested salt. A high sodium and low potassium intake may contribute to BP elevation. Meta-analyses and clinical studies have shown that potassium and magnesium supplementation lowers BP and improves lipid metabolism $(12,20)$. In our precise balance study (28), we demonstrated that replacing ordinary seasonings with seasonings prepared with mineral salt containing a reduced level of sodium and high levels of potassium and magnesium mildly decreased the 24-h urinary sodium excretion $(-21 \%)$ and substantially increased the 24-h urinary potassium $(+55 \%)$ and magnesium excretion $(+22 \%)$. The effects of this combination were larger than expected on the basis of previous estimates, and simultaneous modification of sodium, potassium, and magnesium intake may have a greater impact on BP than a change in one mineral alone for the nonpharmacological treatment of mild hypertension.

The blind sensory taste tests showed that the mineral salt used in these studies is as tasty, palatable, and savory as the seasonings used in Japan. It is commonly accepted that once salt intake has been reduced for a month or more, highly salted foods become distasteful. If salt alternatives are used not only instead of table salt, 'soy sauce' or 'miso', which are traditional Japanese seasonings, but also in processed foods, such as bread, which is one of the largest single sources of salt in the diet, then sodium intake may decrease by another $20 \%$ without a noticeable change in taste. In addition, everyone can also receive a sufficient intake of potassium and magnesium without any special effort. These new seasonings can easily be incorporated into the diet to aid in the management of hypertension and other cardiovascular-related conditions, such as ischemic heart disease and diabetes mellitus.

In conclusion, the Japanese diet, characterized by a high salt intake and an insufficient intake of potassium and magnesium, can thus be easily and safely modified by replacing currently used seasonings prepared with regular salt with those prepared with mineral salt. A reduction in sodium intake coupled with increased intake of potassium and magnesium is likely to contribute to the management of hypertension. A high salt intake may also have a role in bone demineralization (osteoporosis) (43), since there is evidence that the short-term increases in sodium intake can promote urinary calcium excretion and bone turnover. Therefore, the long-term benefits of a reduction in salt intake combined with increased intake of potassium and magnesium may be more important than previously predicted.

\section{Acknowledgements}

We are grateful to the suppliers of the mineral salt (Pansalt ${ }^{\circledR}$ ) used in the seasonings, obtained from Time Associates Co., Ltd., Tokyo, Japan. We also thank Miss 
Asuka Notomi and Miss Miho Sanefuji for their valuable technical assistance.

\section{References}

1. Kempner W: Treatment of hypertensive vascular disease with rice diet. Am J Med 1948; 4: 545-577.

2. Kawasaki T, Delea CS, Bartter FC, Smith H: The effect of high-sodium and low-sodium intakes on blood pressure and other related variables in human subjects with idiopathic hypertension. Am J Med 1978; 64: 193-198.

3. Kawasaki T, Ueno M, Uezono $\mathrm{K}$, et al: Salt intake and hypertension. Jpn Circ J 1981; 45: 810-816.

4. MacGregor GA, Markandu ND, Best FE: Doubleblind crossover trial of moderate sodium restriction in essential hypertension. Lancet 1982; i: 351-356.

5. Intersalt Cooperative Research Group: Intersalt: An international study of electrolyte excretion and blood pressure: results for 24 hour urinary sodium and potassium excretion. $\mathrm{Br}$ Med $J$ 1988; 297: 319-328.

6. Elliott P: Observational studies salt and blood pressure. Hypertension 1991; 17 (Suppl 1): 3-8.

7. Law MR, Frost CD, Wald NJ: By how much does dietary salt restriction lower blood pressure?: analysis of data trial of salt reduction. $B r$ Med $J$ 1991; 302: 819-824.

8. Cutler JA, Follmann D, Elliott P, Suh IL: An overview of randomized trials of sodium reduction and blood pressure. Hypertension 1991; 17 (Suppl I): I27-I-33.

9. Dyer AR, Shipley M, Elliott P, for the INTERSALT Cooperative Research Group: Urinary electrolyte excretion in 24 hours and blood pressure in the INTERSALT study: I. Estimates of reliability. Am J Epidemiol 1994; 139: 927-939.

10. Rikimaru T, Fujita Y, Okuda T, et al: Responses of sodium balance, blood pressure, and other variables to sodium loading in Papua New Guinea highlanders. Am J Clin Nutr 1988; 47: 502-508.

11. Kawasaki $\mathrm{T}$, Itoh $\mathrm{K}$, Uezono $\mathrm{K}$, et al: Investigation of high salt intake in a Nepalese population with low blood pressure. J Hum Hypertens 1993; 7: 131-140.

12. Whelton PK, He J, Cutler JA, et al: Effects of oral potassium on blood pressure: meta-analysis of randomized controlled trials. JAMA 1997; 277: 16241632.

13. Yamori Y, Kihara M, Nara Y, et al: Hypertension and diet: multiple regression analysis in a Japanese farming community. Lancet 1981; i: 1204.

14. Kihara M, Fujikawa J, Ohtaka M, et al: Interrelationships between blood pressure, sodium, potassium, serum cholesterol and protein intake in Japanese. Hypertension 1984; 6: 736-742.

15. Lai S, Yuanchang $\mathrm{T}$, Weiling $\mathrm{H}$, et al: Urinary electrolytes and blood pressure in three $\mathrm{Yi}$ farmer populations, China. Hypertension 1989; 13: 22-30.

16. Motoyama T, Sano H, Fukuzaki H: Oral magnesium supplementation in patients with essential hypertension. Hypertension 1989; 13: 227-232.

17. Witteman JCM, Grobbee DE, Derkx FHM, et al: Reduction of blood pressure with oral magnesium supplementation in women with mild to moderate hypertension. Am J Clin Nutr 1994; 60: 129-135.

18. Geleijnse JM, Witteman JCM, Bak AAA, et al: Reduction in blood pressure with a low sodium, high potassium, high magnesium salt in older subjects with mild to moderate hypertension. Br Med J 1994; 309: 436-440.

19. Itoh K, Kawasaki $\mathrm{T}$, Yoshida $\mathrm{H}$, Nakamura $\mathrm{M}$ : Effects of oral magnesium supplementation on blood pressure, serum lipids and urinary catecholamine in healthy young subjects. J Hypertens 1994; 12 (Suppl 3): s86 (Abstract)

20. Itoh K, Kawasaki T, Nakamura M: The effects of oral magnesium supplementation on blood pressure, serum lipids and related variables in apparently healthy Japanese subjects. Br J Nutr 1997; 78: 737-750.

21. Joint National Committee on Detection, Evaluation, and Treatment of High Blood Pressure: The sixth report of the Joint National Committee on Detection, Evaluation, and Treatment of High Blood Pressure (JNC-VI). Arch Intern Med 1997; 157: 2413-2446.

22. Guidelines Sub-committee: 1993 Guidelines for the management of mild hypertension: Memorandum from a World Health Organization/International Society of Hypertension. J Hypertens 1993; 11: 905918.

23. Weinberger MH, Fineberg NS: Sodium and volume sensitivity of blood pressure: age and pressure change over time. Hypertension 1991; 18: 67-71.

24. Karppanen $\mathrm{H}$, Tanskanen A, Tuomilehto J, et al: Safety and effects of potassium- and magnesium-containing low sodium salt mixtures. J Cardiovasc Pharmacol 1984; 6: S236-S243.

25. Mervaala EMA, Himberg JJ, Laakso J, Tuomainen $\mathrm{P}$, Karppanen $\mathrm{H}$ : Beneficial effects of a potassiumand magnesium-enriched salt alternative. Hypertension 1992; 19: 535-540.

26. Goh R, Saito T, Arase T, Higuchi S: A comparison of intra-arterial, oscillometric and auscultatory measurements of blood pressure: influence of blood pressure level and arteriosclerosis. Masui 1988; 37: 189196 (Japanese with English Abstract).

27. Kawasaki T, Itoh K, Uezono K, Sasaki H: A simple method for estimating $24 \mathrm{~h}$ urinary sodium and potassium excretion from second morning voiding urine specimen in adults. Clin Exp Pharmacol Physiol 1993; 20: 7-14.

28. Friedewald WT, Levy RI, Fredrickson DS: Estimation of concentration of low-density lipoprotein cholesterol in plasma without use of the preparative ultracentrifuge. Clin Chem 1972; 18: 499.

29. Itoh K, Kawasaki T: Clinical and nutritional study of low sodium, high potassium and high magnesium salt on sensory test and electrolyte balance. Jpn J Nutr 1997; 55: 263-272 (Japanese with English Abstract).

30. Gilleran G, O'leary M, Bartlett WA, et al: Effects of dietary sodium substitution with potassium and magnesium in hypertensive Type II diabetics: a randomized blind controlled parallel study. J Hum Hypertens 1996; 10: 517-521.

31. De la Sierra A, Del mar Lluch M, Coca A, et al: Fluid, ionic and hormonal changes induced by high salt intake in salt-sensitive and salt-resistant hypertensive patients. Clin Sci 1996; 91: 155-161.

32. Altura BM, Altura BT: Role of magnesium in the pathogenesis of hypertension: relationship to its action on cardiac, vascular smooth muscle, and endothelial cells updated. in Laragh $\mathrm{JH}$, Brenner BM (eds): Hypertension: Pathophysiology, Diagnosis, and Management, Vol I, 2nd ed, New York, Raven Press, 1995, pp1213-1242.

33. MacGregor GA, Markandu ND, Best FE, et al: Double-blind randomised crossover trial of moderate sodium restriction in essential hypertension. Lancet 1982; i: 351-355.

34. Stamler R, Stamler J, Gosch FC, et al: Primary prevention of hypertension by nutritional-hygienic means: final report of a randomized, controlled trial. JAMA 1989; 262: 1801-1807.

35. The Trials of Hypertension Prevention Collaborative 
Research Group: The effects of nonpharmacologic interventions on blood pressure of persons with high normal levels: results of the trials of hypertension prevention, Phase I. JAMA 1992; 267: 1213-1220.

36. Cappuccio FP, MarKandu ND, Carney C, Sagnella GA, MacGregor GA: Double-blind randomised trial of modest salt restriction in older people. Lancet 1997; ii: 850-854.

37. Graudal NA, Galloe AM, Garred P: Effects of sodium restriction on blood pressure, renin, aldosterone, catecholamines, cholesterols, and triglyceride: a meta-analysis. JAMA 1998; 279: 1383-1391.

38. Davis WH, Leary WP, Reyes AJ, Olhaberry JV: Mono-therapy with magnesium increases abnormally low high density lipoprotein cholesterol: a clinical assay. Curr Ther Res Clin Exp 1984; 36: 341-346.

39. Parkin SM, Walker K, Ashby P, Robinson DS: Effects of glucose and insulin on the activation of lipoprotin lipase and on protein-synthesis in rat adi- pose tissue. Biochem J 1980; 188: 193-199.

40. Itoh K, Kawasaki T, Uezono K, Sasaki H: Effects of high potassium diet on blood pressure and lipid metabolism in mild hypertensive subjects. Jpn J Hypertens 1988; A11: 737 (Abstract)

41. Rasmussen HS, Aurup P, Goransson L, et al: Magnesium deficiency in patients with ischemic heart disease, with and without acute myocardial infarction, uncovered by an intravenous loading test. Arch Intern Med 1988; 148: 329-332.

42. Rasmussen HS, Aurup P, Goldstein K, et al: Influence of magnesium substitution therapy on blood lipid composition in patients with ischemic heart disease. Arch Intern Med 1989; 149: 1050-1053.

43. MacGregor GA, Sever PS, conveners of CASH (Consensus Action on Salt and Hypertension): Saltoverwhelming evidence but still no action: can a consensus be reached with the food industry? $\mathrm{Br} \mathrm{Med} \mathrm{J}$ 1996; 312: 1287-1289. 